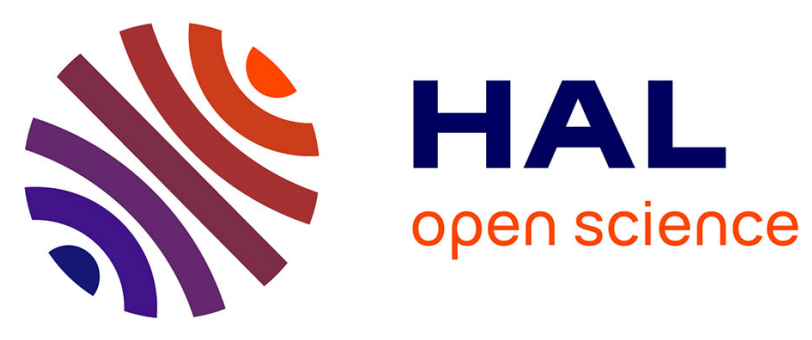

\title{
Impact of the Geometry, Path-Loss Exponent and Random Shadowing on the Mean Interference Factor in Wireless Cellular Networks
}

Bartlomiej Blaszczyszyn, Mohamed Kadhem Karray, Francois-Xavier Klepper

\section{- To cite this version:}

Bartlomiej Blaszczyszyn, Mohamed Kadhem Karray, Francois-Xavier Klepper. Impact of the Geometry, Path-Loss Exponent and Random Shadowing on the Mean Interference Factor in Wireless Cellular Networks. Third Joint IFIP Wireless and Mobile Networking Conference (WMNC), Obuda University and Scientific Association for Infocommunications Hungary, Budapest University of Technology and Economics., Oct 2010, Budapest, Hungary. 10.1109/WMNC.2010.5678764 . inria-00498485v2

\section{HAL Id: inria-00498485 https://hal.inria.fr/inria-00498485v2}

Submitted on 8 Jul 2010

HAL is a multi-disciplinary open access archive for the deposit and dissemination of scientific research documents, whether they are published or not. The documents may come from teaching and research institutions in France or abroad, or from public or private research centers.
L'archive ouverte pluridisciplinaire HAL, est destinée au dépôt et à la diffusion de documents scientifiques de niveau recherche, publiés ou non, émanant des établissements d'enseignement et de recherche français ou étrangers, des laboratoires publics ou privés. 


\section{Impact of the Geometry, Path-Loss Exponent and Random Shadowing on the Mean Interference Factor in Wireless Cellular Networks}

\author{
Bartłomiej Błaszczyszyn \\ INRIA-ENS and \\ Math. Inst. Univ. Wrocław \\ 23 Avenue d'Italie \\ 75214 Paris, France \\ Bartek.Blaszczyszyn@ens.fr
}

\author{
Mohamed Kadhem Karray \\ France Telecom, R\&D Division \\ 38/40 rue Général Leclerc, \\ 92794 Issy-les-Moulineaux France \\ mohamed.karray@orange-ftgroup.com
}

\author{
François-Xavier Klepper \\ INRIA-ENS \\ 23 Avenue d'Italie \\ 75214 Paris, France \\ Francois-Xavier.Klepper@inria.fr
}

\begin{abstract}
The interference factor, defined for a given location in the network as the ratio of the sum of the path-gains form interfering base-stations (BS) to the path-gain from the serving BS is an important ingredient in the analysis of wireless cellular networks. It depends on the geometric placement of the BS in the network and the propagation gains between these stations and the given location. In this paper we study the mean interference factor taking into account the impact of these two elements. Regarding the geometry, we consider both the perfect hexagonal grid of BS and completely random Poisson pattern of BS. Regarding the signal propagation model, we consider not only a deterministic, signal-power-loss function that depends only on the distance between a transmitter and a receiver, and is mainly characterized by the so called path-loss exponent, but also random shadowing that characterizes in a statistical manner the way various obstacles on a given path modify this deterministic function. We present a detailed analysis of the impact of the path loss exponent, variance of the shadowing and the size of the network on the mean interference factor in the case of hexagonal and Poisson network architectures. We observe, as commonly expected, that small and moderate shadowing has a negative impact on regular networks as it increases the mean interference factor. However, as pointed out in the seminal paper [16], this impact can be largely reduced if the serving $\mathrm{BS}$ is chosen as the one which offers the smallest path-loss. Revisiting the model studied in this latter paper, we obtain a perhaps more surprising result saying that in large irregular (Poisson) networks the shadowing does not impact at all the interference factor, whose mean can be evaluated explicitly in a simple expression depending only on the path-loss exponent. Moreover, in small and moderate size networks, a very strong variability of the shadowing can be even beneficial in both hexagonal and Poisson networks.

Index Terms-Wireless Cellular Networks, Interference, Shadowing, f-Factor, Geometry, Poisson, Honeycomb.
\end{abstract}

\section{INTRODUCTION}

The so-called interference factor, defined as the ratio of the interference to the signal power received at a given location, when all the base stations transmit the same power is a keyingredient in the analysis of the quality of service (QoS) perceived at the given location in wireless cellular networks. Its pertinence is by far not limited to the constant power scenario. For example, it appears in the analysis of power and bandwidth allocation problem for broadband systems, which use either Code-Division Multiple Access (CDMA) or Orthogonal Frequency-Division Multiple Access (OFDMA). Moreover, it has been shown that the QoS perceived by the users, measured in terms of blocking probability, mean delay and throughput may be evaluated analytically by using appropriate queueing models, whose key elements are interference factors of users. This means that the study of the field of interference factor (indexed by different locations in the network) gives a primary insight into the performance of wireless cellular networks and thus can be helpful for the capacity planning and dimensioning of these networks.

Two ingredients: the geometric (or geographic) locations of the base-stations (BS) and the propagation losses between these stations and a given location in the network entirely define the interference factor at this location. Regarding the impact of the geometry, it is known that in regular network architectures, in which BS are well receded from each other, one observes smaller (desired) interference factors than in less regular networks, which exhibit some clustering of BS. Mathematical models of hexagonal network and random Poisson repartition of BS are two "extremal" examples of a very regular and completely irregular architectures, respectively. The above general observations regarding the impact of the network geometry on the interference factor can be modified by some particular character of the propagation loss in the network.

The propagation loss comprises the effect of the decay of the received power with the distance (existing even in the free space propagation models) and that of various obstacles between transmitters and receivers (trees, buildings, hills, etc) present in real network profiles. Complex character of various possible network profiles makes pertinent statistical modeling of the propagation loss. In this approach, the propagation loss is typically modeled by the product of a deterministic function of the distance, which represents average path-loss on the given distance in the network, and a random variable, called shadowing, that takes into account in a statistical manner the deviation from this average observed for each particular pair of emitter and receiver. The deterministic path loss function is commonly assumed to be some power of the distance, with the exponent called path-loss exponent. While, the random shadowing variable is often assumed to have log-normal distribution, normalized to have mean one and parametrized by its variance or standard deviation.

In this paper we study jointly the impact of the network geometry and the propagation loss, and in particular of the variance of the shadowing on the mean interference factor (av- 
eraged over possible locations in the network and shadowing scenarios). Our main findings are as follows.

- In regular (hexagonal) networks small and moderate (variance of the) shadowing, as commonly expected, increases the mean interference factor. However, large shadowing can be beneficial as it decreases the mean interference factor, asymptotically making it even close to zero.

- In irregular (Poisson) network even small shadowing can be beneficial.

- Strong shadowing makes the value of the mean interference factor in regular network comparable to this of irregular one. In this case one can say that the impact of the shadowing completely "dominates" this of the geometry.

- Increasing the size of the network in general increases the interference factor, however, this increase is negligible for higher path loss exponent and small and moderate shadowing. For this regime infinite network models may be reasonably used.

- The distribution of the interference factor in the infinite Poisson network model does not depend on the distribution of the shadowing and its mean is equal to $2 /(\beta-2)$, where $\beta$ is the path-loss exponent.

- The mean of the interference factor in the infinite hexagonal model is not known in an explicit form, however it is conjectured to increase with the variance of the shadowing asymptotically to some non-zero and finite value smaller or equal to this of Poisson network.

The remaining part of this paper is organized as follows. In the next section we briefly present related works. In Section III we describe our models. The main results obtained by simulations of these models are presented in Section IV. Next, in Section $\mathrm{V}$ we present mathematical analysis of the models, which supports and completes our numerical findings. Finally, in Section VI we provide some concluding remarks.

\section{RELATED WORKS}

The interference factor was recognized very early as a key element in the performance evaluation of cellular networks; cf. [17], [10]. More recently in [3], [5], [6] it appeared in the analysis of the CDMA, HSDPA and OFDMA; cf also [2, Chapter 19]. It is often computed by simulations (see for example [15]). Some papers (see e.g. [9], [7]) propose more explicit approximations of the interference factor and its moments (mean and variance) assuming only deterministic propagation loss models (without random shadowing). [13] studies the distribution of the interference factor in such a case.

Our propagation loss model is commonly accepted in the literature; see e.g. [14] where log-normal shadowing of mean 1 is considered. A possible extension of this model consists in assuming shadowing distribution (say, its variance) that depends on the distance, cf. [11].

The impact of the shadowing on the distribution of the interference factor is studied numerically in [12] and analytically in [8]. However, the above two articles do not take into account the modification of the network geometry induced by the shadowing; i.e., assume that mobiles are served by their geographically closest BS. This is not a realistic assumption and, as we will show in this paper, leads to misleading conclusions that the shadowing dramatically increases the mean interference factor.

The paper [16] focuses on the interference factor averaged over a given cell, and in particular the effect of shadowing on this average. It is shown there that the cell shape modification induced by the shadowing affects significantly the mean interference factor. More precisely, that this mean decreases substantially if mobiles are served by the BS offering the smallest path-loss. We adopt this assumption throughout the present paper in the context of regular (hexagonal) and irregular (Poisson) geometry of BS, as proposed in [3].

\section{MODEL DESCRIPTION}

\section{A. Location of base stations}

In this paper we will consider two particular models for the location of BS, hexagonal and Poisson one. The former is commonly considered as an "ideal" model for the cellular networks, while the latter one can be seen as an extremal case of very irregular network.

1) Infinite Models:

- Hexagonal network. Consider BS located on a regular hexagonal grid on $\mathbb{R}^{2}$ with the distance $\Delta$ between two adjacent vertexes of this grid ${ }^{1}$; cf. Figure 1 . Note that the surface area of a given cell (hexagon; i.e., subset of the plane whose points are closer to a given point of the grid than to any other) of this model is equal to $\sqrt{3} \Delta^{2} / 2$. Thus the intensity of the BS in this model is equal to $\lambda=2 /\left(\sqrt{3} \Delta^{2}\right) \mathrm{BS} / \mathrm{km}^{2}$. In what follows it will be customary to consider a stationary version $\Phi_{H}$ of this grid, which can be obtained by randomly shifting it through a vector uniformly distributed in one given hexagon (cf [1, Example 4.2.5]). In this model a given location, say the origin of the plane, corresponds to an "arbitrary" location of a mobile, "randomly chosen" in the network.

- Poisson network. Assume that BS are located at the points of a stationary, homogeneous Poisson point process (p.p.) $\Phi_{P}$ of intensity $\lambda \mathrm{BS} / \mathrm{km}^{2}$ on the plane $\mathbb{R}^{2}$. When comparing performance of Poisson and hexagonal model we will always take them with the same intensity $\lambda=2 /\left(\sqrt{3} \Delta^{2}\right)$.

Considering infinite models is often a convenient way of studying phenomena arising in very large networks. A particular property of these models is lack of (geographic) boundary effects, which in real, large but finite, networks, have often a negligible impact on performance characteristics measured in the "middle" of the network. However, as we will see in this paper, sometimes mathematical assumption of an infinite network may create some artifacts, which are not observed in more realistic, large but finite, networks.

\footnotetext{
${ }^{1}$ The set of vertexes of this grid can be described on the complex plane by $\left\{\Delta\left(u_{1}+u_{2} e^{i \pi / 3}\right), u=\left(u_{1}, u_{2}\right) \in\{0, \pm 1, \ldots\}^{2}\right\}$.
} 


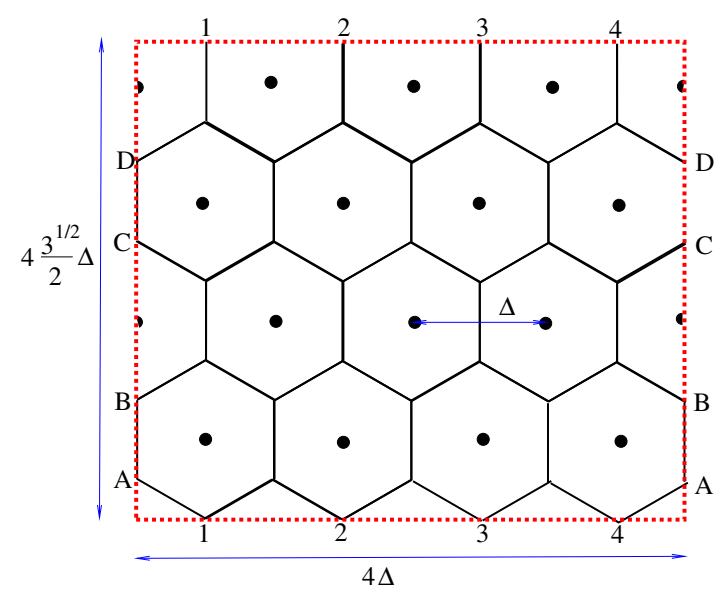

Fig. 1. hexagonal pattern of $4 \times 4 \mathrm{BS}$ on rectangular torus $\mathbb{T}_{4}$. Identified points are denoted by the same digits or characters.

2) Bounded Models: In order to have finite network models, and still neglect the boundary effects (which might be reasonable for large networks) one often considers toroidal model. Recall that, roughly speaking, rectangular torus is a rectangle whose opposite sites are "identified". For $N=2,4,6, \ldots$, we will denote by $\mathbb{T}_{N}$ the rectangle $[-N \Delta / 2, N \Delta / 2) \times$ $[-N \sqrt{3} \Delta / 4, N \sqrt{3} \Delta / 4)$ with toroidal metric. Restricting $\Phi_{H}$ to $\mathbb{T}_{N}$, i.e. taking $\Phi_{H}^{\mathbb{T}}=\Phi_{H} \cap \mathbb{T}_{N}$ one obtains the model whose distribution is invariant with respect to translations on the torus. Thus we obtain a hexagonal network model that consists of $N^{2}$ cells (cf Figure 1) and which does not exhibit any border effects. Similarly we will consider the restriction $\Phi_{P}^{\mathbb{T}}$ of the Poisson p.p. $\Phi_{P}$ to $\mathbb{T}_{N}$.

\section{B. Path-loss model with shadowing}

For a given BS $X \in \Phi\left(\Phi=\Phi_{P}\right.$ or $\left.\Phi_{H}\right)$ and a given location $y \in \mathbb{R}^{2}$ on the plane we denote by $L_{X}(y)$ the (timeaverage, i.e., averaged out over the fading) propagation-loss between BS $X$ and location $y$. In what follows we will always assume that

$$
L_{X}(y)=\frac{L(|X-y|)}{S_{X}(y)},
$$

where $L(\cdot)$ is a non-decreasing, deterministic function of the distance between an emitter and a receiver, and $S_{X}(\cdot)$ is a random shadowing field related to the $\mathrm{BS} X$. In what follows we will always assume that given locations of BS $\left\{X_{i} \in \Phi\right\}$ their shadowing fields $\left\{S_{X_{i}}(\cdot)\right\}$ are independent non-negative stochastic processes, each being indexed by locations $y \in \mathbb{R}^{2}$. More formally speaking, the locations of BS $X$ and their respective shadowing fields $S_{X}(\cdot)$ form an independently marked version $\tilde{\Phi}=\left\{\left(X, S_{X}(\cdot)\right)\right\}_{X \in \Phi}$ of the point process $\Phi$.

Regarding the distribution of the marks (shadowing fields) of this process, they are assumed to have the same marginal distributions; i.e., given $X, S_{X}(y)$ has the same distribution for all $y \in \mathbb{R}^{2}$, of normalized mean $\mathbf{E}\left[S_{X}(y)\right]=1$, with the following two cases being of particular interest

- $S_{X}(y) \equiv 1$, which corresponds to a case with negligible shadowing (we will say also "no shadowing"),

- for all $y, S_{X}(y)$ is log-nornal random variable with mean 1. Recall that such a mean-1 log-normal variable
$S$ can be expressed as $S=e^{\mu+\sigma N}$ where $N$ is standard Gaussian random variable (with mean 0 and variance 1) with $\mu=-\sigma^{2} / 2$. Indeed, in this case $\mathbf{E}[S]=e^{\mu+\sigma^{2} / 2}=$ 1. Note that if the shadowing is log-normal random variable then the path-loss (at a given distance) expressed in $\mathrm{dB}$ is Gaussian random variable. Furthermore, in this context it is common to parametrize the log-normal shadowing by the standard deviation (SD) of $S$ expressed in $\mathrm{dB}$; i.e., the $\mathrm{SD}$ of $10 \log _{10} S$. We will denote it by $v$. With respect to the previous parametrisation we have $v=\sigma 10 / \log 10$. Throughout the paper we will call $v$ the logarithmic standard deviation $(\log -S D)$ of the shadowing.

In this paper we do not need to make any particular assumption on the correlation of the shadowing field $S_{X}(y)$ for given $X$ and different locations $y$. Throughout the paper we will implicitly assume also that mean path-gain is finite, i.e., $\mathbf{E}[1 / S]<\infty$. Note that this condition is satisfied for log-normal variable, indeed, in our case of mean-1 variable $\mathbf{E}[1 / S]=e^{\sigma^{2}}=e^{v^{2} \log ^{2} 10 / 100}$.

For the deterministic path-loss function $L(\cdot)$ the following particular model is often used and will be our default assumption in this paper:

$$
L(r)=(K r)^{\beta}
$$

where $K>0$ and $\beta>2$ are some constants.

\section{Handover policy}

In what follows we will assume that each given location $y \in \mathbb{R}^{2}$ is served by the BS $X_{y}^{*} \in \Phi$ with respect to which it has the weakest path-loss (so, in other words, the strongest received signal, given all $\mathrm{BS}$ emit with the same power), i.e, such that

$$
L_{X_{y}^{*}}(y) \leq L_{X}(y) \quad \text { for all } X \in \Phi,
$$

with any tie-breaking rule. Note that in the case of negligible shadowing $\left(S_{X}(y) \equiv 1\right)$ and strictly increasing function $L(\cdot)$ the above policy corresponds to the geographically closest BS. Note also that for our infinite network models with random shadowing, one has to prove that the minimum of the path-loss is achieved for some BS, i.e.; that $X_{y}^{*}$ is well defined.

\section{Interference factor}

For a given location $y \in \mathbb{R}^{2}$ we define the interference factor $f(y)$ as

$$
f(y)=f(y, \tilde{\Phi})=\sum_{X \in \Phi, X \neq X_{y}^{*}} \frac{L_{X_{y}^{*}}(y)}{L_{X}(y)}=\sum_{X \in \Phi} \frac{L_{X_{y}^{*}}(y)}{L_{X}(y)}-1
$$

provided $X_{y}^{*}$ is well defined. In the remaining part of this paper we will be interested in the expected value $\mathbf{E}[f(y)]=$ $\mathbf{E}[f(y, \tilde{\Phi})]$. By the translation invariance of the distribution of our infinite and toroidal models this expectation 
does not depend on the location and thus, for these models $\mathbf{E}[f(y)]=\mathbf{E}[f(0)]$. $^{2}$

In the remaining part of the paper we will study the impact of the shadowing and also the geometry and size of the network on the mean inference factor $\mathbf{E}[f(0)]$. Note by the homothetic invariance of our hexagonal and Poisson models with the path-loss function (2), the mean interference factor in the infinite models does not depend on the intensity $\lambda$ of BS and in toroidal models does not depend on the parameter $\Delta$ (but only on the size $N$ of the network).

\section{NumeriCAL RESUlts}

In this section we will consider Poisson and hexagonal models on the torus $\mathbb{T}_{N}$ with log-normal shadowing. We estimate the value of $\mathbf{E}[f(0)]$ by simulations. Figures 2 and 3 show the impact of the path-loss exponent, shadowing and the size of the network in the case of the hexagonal and Poisson network architecture, respectively. Here are our main observations.

Remark 4.1: 1) Observe on Figure 2 for hexagonal network of a given size $N^{2}$ BS, with $N=6,10,30$, and a given path-loss exponent $\beta=3,4,5$, that the mean interference factor $\mathbf{E}[f(0)]$ first increases and then decreases to 0 when the value $v$ of logarithmic standard deviation $(\log -D S)$ of the shadowing increases.

2) or the Poisson network (see Figure 3) $\mathbf{E}[f(0)]$ is decreases in log-SD starting already from very small values of $v$.

3) The actual size of the network consisting of $N^{2} B S$, when $N \geq 100$, has negligible impact on $\mathbf{E}[f(0)]$ when $\beta=4$ and $v \leq 10$ or $\beta=5$ and $v \leq 15$ both in hexagonal and Poisson case (in this latter case $N^{2}$ is the expected number of BS). In this regime the value of $\mathbf{E}[f(0)]$ corresponds to this in the respective infinite model. In particular, for Poisson network it is equal to $2 /(\beta-2)$ and does not depend on log-SD $v$ (cf Proposition 5.5 below).

4) When $\beta=4$ and $v \geq 10$ or $\beta=5$ and $v \geq 15$ the mean interference factor $\mathbf{E}[f(0)]$ non-negligibly increases with the network size.

5) Comparing Figures 2 and 3 for $v \geq 20$ we observe that for large log-SD of the shadowing the mean interference factor evaluated for the Poisson network is almost exactly the same as for the hexagonal network of the same size.

Remark 4.2: The seminal paper [16] considers only the hexagonal network architecture, however, the beneficial impact of the shadowing is not observed there. The reason is that the model considered in [16] assumes that the smallest-path-loss BS (the serving one) is selected among the $N_{C}$ closest BS.

\footnotetext{
${ }^{2}$ Often the mathematical expectation $\underset{\tilde{\Phi}}{\mathbf{E}}[f(0, \tilde{\Phi})]$ corresponds to the empirical mean value $\lim _{n \rightarrow \infty} 1 / n \sum f\left(y_{i}, \tilde{\Phi}\right)$ of the interference factor measured at many locations "uniformly" sampled in one given realization of the network and shadowing. A precise statement and rigorous proof of such an ergodic result is beyond the scope of this paper. We remark only that for the hexagon network on the torus, this result follows simply form the Law of Large Numbers, when $y_{i}$ are independently and uniformly distributed and provided the shadowing variables $S_{X}\left(y_{i}\right)$ are independent across different values of $y_{i}$. This latter assumption, corresponding to spatially uncorrelated shadowing, is not made in this paper since it is not needed for other results.
}

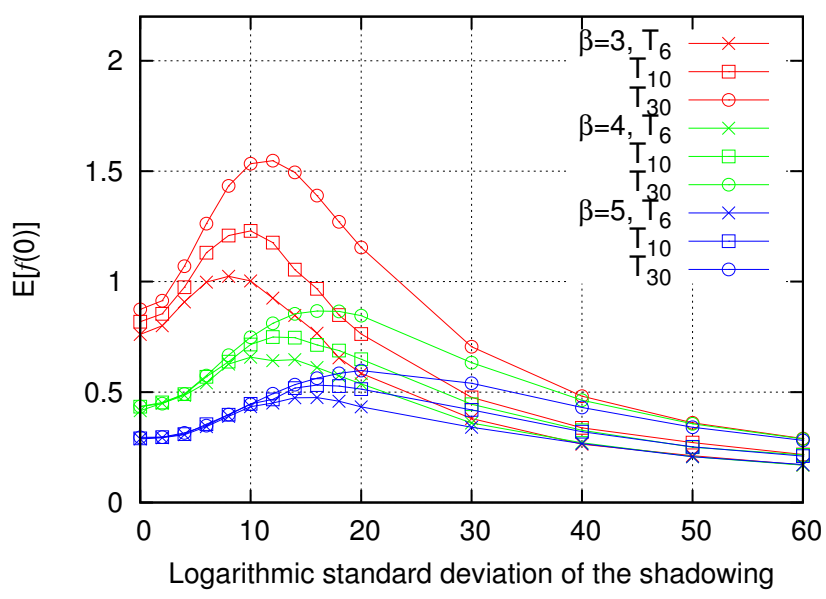

Fig. 2. Mean interference factor in hexagonal network on the torus $\mathbb{T}_{N}$ with log-normal shadowing with $\log$-SD $v$ and path-loss exponent $\beta$.

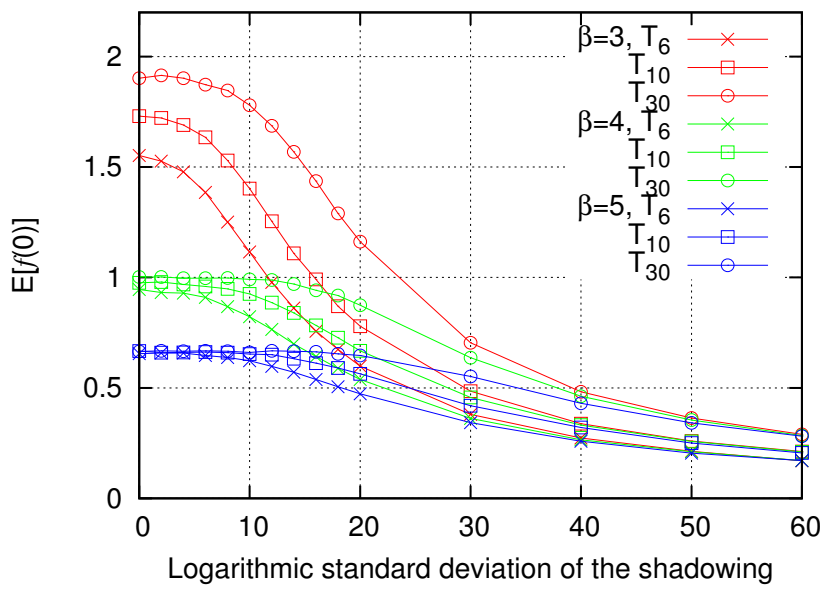

Fig. 3. Mean interference factor in Poisson network on the torus $\mathbb{T}_{N}$ with $\log$-normal shadowing with $\log$-SD $v$ and path-loss exponent $\beta$.

In particular, $N_{C}=1$ ignores the shadowing in the hand-over policy as it corresponds to the situation where the serving BS is always the closest one. On the other hand the model considered in our paper corresponds to $N_{C}$ equal to the total number of BS in the network. In consequence, for a higher path-loss exponent (say $\beta=4$ ) and small and moderate logSD of the shadowing $(0 \leq v \leq 12)$ our numerical results are close to these of [16] with $N_{C}=4$; cf. our Figure 2 and the last column in Table 1 in [16]. The fact that the average interference factor decreases in some cases with logSD of the shadowing has not been observed in [16] due to the set of parameters considered there. Indeed, for a smaller path-loss exponent, $\beta=3$, our Figure 2 shows the mean interference factor decreasing in $v$ starting from $v \approx 8$. This range of parameters is also considered in [16, Table 2] however, with the $N_{C}=2$. Apparently the beneficial impact of the shadowing cannot be observed in this case, when the BS can be chosen only among two closest BS. A general remark is of the following order: strong shadowing requires larger geographical domain in which the serving BS is searched, as the optimal one may be located far from the mobile. 
In the remaining part of the paper we will state and prove some mathematical results regarding $\mathbf{E}[f(0)]$, which support and extend the above numerical findings.

\section{Mathematical Results}

\section{A. Interference factor in toroidal models}

The somewhat surprising observation regarding the beneficial impact of the strong log-SD of the shadowing on the mean interference factor can be confirmed mathematically.

Proposition 5.1: Assume an arbitrary, fixed, finite pattern $\left\{X_{1}, X_{2}, \ldots, X_{n}\right\}$ of BS locations. Consider any deterministic path-loss function $0<L(r)<\infty$ and (independent) lognormal shadowing $S_{X_{i}}(\cdot)$ with the $\log -S D$. Then for any location $y$ we have $\lim _{v \rightarrow \infty} f(y)=0$ in probability.

Proof: We will show that $\lim _{v \rightarrow \infty} \operatorname{Pr}\{f(y) \geq \epsilon\}=0$ for any $\epsilon>0$. Denote by $G_{i}=S_{X_{i}}(y) / L\left(\left|X_{i}-y\right|\right)$ the pathgain from $X_{i}$ to $y$. Consider ordered vector $\left(G_{(1)}, \ldots, G_{(n)}\right)$ of these path gains, where $\min _{i} G_{i}=G_{(1)} \leq \ldots \leq$ $G_{(n)}=\max _{i} G_{i}$. Note that $f(y)=1 / G_{(n)} \sum_{i=1}^{n} G_{(i)}-$ $1 \leq(n-1) G_{(n-1)} / G_{(n)}$. In order to prove our claim it is enough to show that $\operatorname{Pr}\left\{G_{(n-1)} / G_{(n)} \geq \epsilon\right\} \rightarrow 0$ when $v \rightarrow \infty$. To this regard denote $L\left(\left|X_{i}-y\right|\right)=l_{i}$, and recall from the definition of our path-loss model that we can represent $G_{i}(y)=e^{\tilde{N}_{i}}$, where $\left\{\tilde{N}_{i}\right\}_{i=1, \ldots, n}$ are independent Gaussian random variables, with mean $\mathbf{E}\left[\tilde{N}_{i}\right]=$ $-\log l_{i}-\sigma^{2} / 2$ and the same SD $\sigma=v \log 10 / 10$. Since $G_{i}$ is monotone increasing in $\tilde{N}_{i}$ we have $G_{(i)}=e^{\tilde{N}_{(i)}}$, where $\min _{i} \tilde{N}_{i}=\tilde{N}_{(1)} \leq \ldots \leq \tilde{N}_{(n)}=\max _{i} \tilde{N}_{i}$. Moreover, $A:=\left\{G_{(n-1)} / G_{(n)} \geq \epsilon\right\}=\left\{\tilde{N}_{(n)}-\tilde{N}_{(n-1)} \leq M\right\}$, where $M=-\log \epsilon$. Denote by $A_{i j}=\left\{0 \leq \tilde{N}_{i}-\tilde{N}_{j} \leq M\right\}$. Note that $A \subset \bigcup_{i, j=1, \ldots, n, i \neq j} A_{i j}$ and the result follows from the fact that for any $i \neq j \operatorname{Pr}\left\{A_{i j}\right\} \rightarrow 0$ when $v \rightarrow \infty$. Indeed, for $i \neq j, \tilde{N}_{i}-\tilde{N}_{j}=\bar{N}$ is Gaussian random variable with mean $\log \left(l_{j} / l_{i}\right)$ and variance $\sigma^{2}$ and thus $\operatorname{Pr}\left\{A_{i j}\right\}=\operatorname{Pr}\{0 \leq \bar{N} \leq M\} \rightarrow 0$ for any given finite $M$ when $\sigma^{2}=v^{2} \log ^{2} 10 / 100 \rightarrow \infty$. This completes the proof.

Corollary 5.2: The mean interference factor $f(0)$ in the Poisson and hexagonal network on the torus $\mathbb{T}_{N}$, with lognormal shadowing converges in distribution and in expectation to 0 when $\log$-SD of the shadowing goes to infinity.

Proof: For any $\epsilon>0$ by Proposition 5.1 and Lebesgue dominated convergence theorem we have $\operatorname{Pr}\{f(0, \tilde{\Phi})>\epsilon\}=$ $\mathbf{E}[\operatorname{Pr}\{f(0, \tilde{\Phi})>\epsilon \mid \Phi\}] \rightarrow 0$, when $v \rightarrow \infty$. This proves that $f(0)$ converges in distribution to 0 . Convergence of $\mathbf{E}[f(0)]$ to 0 follows again from the Lebesgue dominated convergence theorem by the observation $f(y, \tilde{\Phi}) \leq \Phi\left(\mathbb{T}_{N}\right)-1$ and $\mathbf{E}\left[\Phi\left(\mathbb{T}_{N}\right)\right]<\infty$.

\section{B. Interference factor - infinite models}

In this section we will consider infinite hexagonal and Poisson models. We will show first that serving BS $X_{0}^{*}$, and hence interference factor, is well defined. Then we will argue that values of this factor in the infinite models can be seen as limits of respective toroidal models on $\mathbb{T}_{N}$ when $n \rightarrow \infty$. Finally we will prove a (surprising ?) invariance of $\mathbf{E}[f(0)]$ in the infinite Poisson model with respect to the distribution of the shadowing. In the case the value $\mathbf{E}[f(0)]$ can be evaluated explicitly.

Proposition 5.3: Consider infinite Poisson $\Phi=\Phi_{P}$ or hexagonal $\Phi=\Phi_{H}$ model of $B S$, with shadowing whose marginal distribution has finite moment of order $2 / \beta\left({ }^{3}\right)$. Then there exist $X_{0}^{*} \in \Phi$ satisfying (3). Moreover, the interference factor calculated with respect to the restriction of $\Phi$ to $\mathbb{T}_{N}$, i.e., $f\left(0, \tilde{\Phi}^{\mathbb{T}_{N}}\right)$, converges almost surely and in expectation to $f(0, \tilde{\Phi})$.

Proof: To prove the first statement it is enough to show that the expected number of BS $X_{i}$ such that $S_{X_{i}}(0) / L\left(\left|X_{i}\right|\right)>M$ is finite for any $M<\infty$. In the case of the Poisson p.p. this will be shown in the proof of Proposition 5.5 below. Here we consider only hexagonal case $\Phi=\Phi_{H}$. Denote by $\bar{G}(x)=\operatorname{Pr}\{S>x\}$. We have

$$
\begin{aligned}
\mathbf{E} & {\left[\#\left\{X_{i} \in \Phi_{H}: S_{X_{i}(0)} / L\left(\left|X_{i}\right|\right)>M\right\}\right] } \\
& =\mathbf{E}\left[\sum_{X_{i} \in \Phi_{H}} \mathbb{1}\left(S_{X_{i}(0)}>M L\left(\left|X_{i}\right|\right)\right)\right] \\
& =\mathbf{E}\left[\sum_{X_{i} \in \Phi_{H}} \bar{G}\left(M L\left(\left|X_{i}\right|\right)\right)\right] \\
& \leq \sum_{i=1}^{\infty} 6 n \bar{G}\left((n \Delta K / 2)^{\beta} / M\right)<\infty,
\end{aligned}
$$

where the last inequality follows from the assumption $\mathbf{E}\left[S^{2 / \beta}\right]=2 / \beta \int_{0}^{\infty} s^{2 / \beta-1} \bar{G}(s) d s<\infty$. This completes the proof of the first statement.

In order to prove the second statement, note that for any realization the network $\tilde{\Phi}$, for $N$ large enough $X_{0}^{*} \in \mathbb{T}_{N}$ and consequently $f\left(0, \tilde{\Phi}^{\mathbb{T}_{N}}\right)$ eventually increases in $N$ (the serving BS is not changing any more and only interference is added). The convergence of expectation follows from the dominated convergence theorem knowing that $f(0, \tilde{\Phi}) \leq f^{\prime}(0, \tilde{\Phi})$, where $f^{\prime}(0, \tilde{\Phi})$ is the interference factor calculated under assumption that the handover policy selects the geographically closest BS as the serving one. By the independence of the shadowing fields given the locations of BS and the assumption that the mean shadowing is equal to 1

$$
E\left[f^{\prime}(0, \tilde{\Phi})\right]=E\left[\frac{1}{S}\right] \mathbf{E}\left[\sum_{X \in \Phi} \frac{L\left(\left|X_{0}^{\prime *}\right|\right)}{L(|X|)}\right]-1,
$$

where $X_{0}^{\prime *}$ is a point of $\Phi$ closest to the origin 0 . By our assumption on the mean path-gain $\mathbf{E}[1 / S]<\infty$. The second expectation (5) is equal to the mean interference factor in the infinite model with constant shadowing $S \equiv 1$, and it is known to be finite in the infinite hexagonal and Poisson model; cf. respectively Remark 5.4 and Proposition 5.5 below.

Remark 5.4: It was shown in [3] that in the case of $S \equiv$ 1 and the deterministic path-loss function (2) $\mathbf{E}[f(0)]$ in the hexagonal model is finite and can be approximated by the expression

$$
\mathbf{E}\left[f\left(0, \Phi_{H}\right)\right] \approx \frac{0.9365}{\beta-2},
$$

which does not depend on the density of BS.

We consider now infinite Poisson model.

3i.e., $\mathbf{E}\left[S^{2 / \beta}\right]<\infty$. Note that $2 / \beta<1$ and thus the above assumption follows from our default assumption $\mathbf{E}[S]=1<\infty$. 
Proposition 5.5: Assume Poisson network, deterministic path-loss function (2). The distribution of the interference factor $f(0)=f(0, \tilde{\Phi})$ does not depend on the marginal distribution of the shadowing field $S_{X}(\cdot)$ provided $\mathbf{E}\left[S^{2 / \beta}\right]<\infty$. Moreover, we have $\mathbf{E}[f(0)]=2 /(\beta-2)$.

Remark 5.6: The above result says that in the infinite Poisson notwork the existence of shadowing (satisfying our model assumptions) has no impact on the interference factor in Poisson network, whose mean has the same value as in this network with negligible shadowing. The expression $2 /(\beta-2)$ has been found in this latter context for the first time (to the best of our knowledge) in [4]. Note however, that the above observation is valid only if the handover policy selects the strongest BS as described in Section III-C. Indeed, assume that, despite non-constant shadowing, the handover policy selects the geographically closest BS as the serving one. Then, the mean interference factor $\mathbf{E}\left[f^{\prime}(0)\right]$ can be expressed as in (5). Recall that the second expectation in this expression is equal to the mean interference factor in the same model without shadowing (i.e., $S \equiv 1$ ). By the Jensen's inequality $\mathbf{E}[1 / S] \geq 1 / \mathbf{E}[S]=1$ and consequently we observe the increase of the mean interference factor with respect to the "shadowing-dependent" handover policy. In particular, for lognormal $S$ with mean 1 and $\log$-SD $v$ we have $\mathbf{E}[1 / S]=e^{\sigma^{2}}=$ $e^{v^{2} \log ^{2} 10 / 100}$, which means that the log-normal shadowing in any geometric model of BS in which it is not taken into account in the handover policy increases the mean interference factor by $v^{2} \log 10 / 10 \mathrm{~dB}$, where $v$ is $\log$-SD of the shadowing.

Proof: (of Proof of Proposition 5.5.) Note that the value of $f(0)$ is entirely defined by the collection of random variables $\left\{L_{X}(0)=L(|X|) / S_{X}(0): X \in \Phi\right\}$. Given $\Phi$ these random variables are independent. Thus by the displacement theorem for Poisson p.p. (cf [1, Theorem 1.3.9]) $\left\{L_{X}(0)\right\}=\Psi$ constitutes a (non-homogeneous) Poisson p.p. on $\mathbb{R}^{+}=[0, \infty)$ of intensity measure $\Lambda^{\prime}$ given by

$$
\begin{aligned}
\Lambda^{\prime}([0, s]) & =\mathbf{E}[\Psi([0, s])] \\
& =\lambda \int_{\mathbb{R}^{2}} \operatorname{Pr}\{L(|z|) / S \leq s\} d z \\
& =2 \pi \lambda \int_{0}^{\infty} r \operatorname{Pr}\{L(r) / S \leq s\} d r \\
& =2 \pi \lambda \int_{0}^{\infty} r \mathbf{E}[\mathbb{1}(L(r) / S \leq s)] d r \\
& =2 \pi \lambda \mathbf{E}\left[\int_{0}^{(s)^{1 / \beta} / K} r d r\right] \\
& =\frac{\pi \lambda s^{\frac{2}{\beta}}}{K^{2}} \mathbf{E}\left[S^{\frac{2}{\beta}}\right] .
\end{aligned}
$$

Note that the latter expression is finite, which proves that the serving BS $X_{0}^{*}$ is well defined (cf proof of Proposition 5.3). Note also that it depends on the shadowing only through its moment $\mathbf{E}\left[S^{2 / \beta}\right]$. Moreover one obtains the same expression in the model without shadowing and the density of BS multiplied by $\mathbf{E}\left[S^{2 / \beta}\right]$. However, by the homothetic invariance of the Poisson model with the path-loss function (2) the distribution of $f(0)$ does not depend on the intensity of the BS. Thus the invariance of the distribution of $f(0)$ on the distribution of the shadowing. In particular, we can conclude that $\mathbf{E}[f(0)]=2 /(\beta-2)-$ the value obtained in the model without shadowing; see [4], cf. also [1, Example 4.5.1]. This completes the proof.

\section{CONCLUDING REMARKS}

Random shadowing is believed to increase the interference factor in the wireless cellular networks. This statement is true and the increase is tremendous (expressed in $\mathrm{dB}$, it is of order of the share of the logarithmic standard deviation of the shadowing) if the handover policy ignores the shadowing in the path-loss to the base stations. However, taking the shadowing into account in the handover policy, allows to completely cancel out its negative impact on the mean interference factor in large irregular (Poisson) networks. Moreover, large variability of the shadowing can be even beneficial for small and medium size hexagonal and Poisson networks. However, these "optimistic" results regard the mean interference factor and not worst-case scenarios. More study, in particular of impact of the shadowing on the distribution of the interference factor is needed.

\section{REFERENCES}

[1] F. Baccelli and B. Błaszczyszyn. Stochastic Geometry and Wireless Networks, Volume I - Theory, volume 3, No 3-4 of Foundations and Trends in Networking. NoW Publishers, 2009.

[2] F. Baccelli and B. Błaszczyszyn. Stochastic Geometry and Wireless Networks, Volume II - Applications, volume 4, No 1-2 of Foundations and Trends in Networking. NoW Publishers, 2009.

[3] F. Baccelli, B. Błaszczyszyn, and M.K. Karray. Up and downlink admission/congestion control and maximal load in large homogeneous CDMA networks. MONET, 9(6), December 2004.

[4] F. Baccelli, B. Błaszczyszyn, and F. Tournois. Downlink admission/congestion control and maximal load in CDMA networks. In Proc. of IEEE Infocom, 2003.

[5] B. Błaszczyszyn and M. K. Karray. Performance evaluation of scalable congestion control schemes for elastic traffic in cellular networks with power control. In Proc. of IEEE INFOCOM, May 2007.

[6] B. Błaszczyszyn and M.K. Karray. Dimensioning of the downlink in OFDMA cellular networks via an Erlang's loss model. In Proc. of European Wireless, 2009.

[7] M.K. Karray. Study of a key factor for performance evaluation of wireless cellular networks: The f-factor. In Proc. of IFIP Wireless Days, December 2009.

[8] J. M. Kelif and M. Coupechoux. Impact of topology and shadowing on the outage probability of cellular networks. In Proc. of IEEE ICC, 2009.

[9] J.M. Kelif, M. Coupechoux, and P. Godlewski. Fluid model of the outage probability in sectored wireless networks. In Proc. of $\mathrm{WCNC}$, pages 2933-2938, 2008.

[10] J.S. Lee and L.E. Miller. CDMA systems engineering handbook. Artech House, Boston, 1998.

[11] Y. Liang, A.J. Goldsmith, G.J. Foschini, R.A. Valenzuela, and D. Chizhik. Evolution of base stations in cellular networks: Denser deployment versus coordination. In Proc. of ICC, 2008.

[12] A. Masmoudi and S. Tabbane. Other-cell-interference factor distribution model in downlink WCDMA systems. In Proc. of MSWiM '04. ACM, 2004.

[13] D. Staehle, K. Leibnitz, K. Heck, B. Schröder, A. Weller, and P. TranGia. Approximating the othercell interference distribution in inhomogeneous UMTS networks. In Proc. of VTC, 2002.

[14] G.L. Stüber. Principle of Mobile Communication. Springer Netherlands, Dordrecht,Netherlands, 2001.

[15] A. Thakur. Outside cell interference dynamics in cellular code division multiple access. PhD thesis, Ohio University, 2003.

[16] A.J. Viterbi, A.M. Viterbi, and E. Zehavi. Other-cell interference in cellular power-controlled CDMA. IEEE Trans. on Comm., 42, March 1994.

[17] A.M. Viterbi and A.J. Viterbi. Erlang capacity of a power controlled CDMA system. IEEE J. Select. Areas Commun., 11(6), August 1993. 